\title{
Controle na alocação de recursos na saúde pública: uma análise nas microrregiões do sudeste brasileiro
}

\section{Control of resource allocations in public healthcare: an analysis in Brazilian southeast micro regions}

Lucas Maia dos Santos

Doutorado em Administração pela Universidade Federal de Minas Gerais. Professor do Instituto Federal de Minas Gerais, Sabará, Brasil, lucas.maia@ifmg.edu.br http://lattes.cnpq.br/6936984179200806

José Roberto de Souza Francisco

Doutorado em Administração e Professor pela Universidade Federal de Minas Gerais, Brasil, j.roberto@ufsj.edu.br http://lattes.cnpq.br/9553851503191616

Márcio Augusto Gonçalves

Doutorado em Administração pela Aston University (Grã-Bretanha). Professor da Universidade Federal de Minas Gerais, Brasil, marciouk@yahoo.com http://lattes.cnpq.br/1295685039791681

Resumo: O presente estudo tem como objetivo investigar a eficiência da alocação de recursos públicos na atenção primária da saúde, tomando como referência as microrregiões do Sudeste do Brasil. Para avaliar a eficiência do setor de saúde, foram selecionadas variáveis que representaram os recursos alocados (inputs) e os serviços oferecidos à sociedade (outputs). A mensuração da eficiência foi realizada por intermédio da Análise Envoltória de Dados, com a utilização do modelo clássico de retornos variáveis com orientação-produto. A fronteira de eficiência obtida não busca fornecer um padrão rígido de eficiência para a saúde, mas uma forma de verificar o quanto as microrregiões poderiam produzir se mantido o atual investimento. Os resultados demonstraram que o desempenho das microrregiões do Sudeste pode ser considerado mediano, e o Estado de São Paulo apresentou o maior número de unidades eficientes. Por fim, o trabalho assevera a importância de utilização de ferramentas de controle baseando-se em resultados para promover políticas para a melhoria da eficiência do atendimento primário em saúde, que consideram as diversidades dos municípios e microrregiões do país, em função das particularidades interregionais.

Palavras-chave: Controle. Saúde Pública. Eficiência. DEA. Gestão Pública.

Abstract: This study aims to investigate the efficiency of resource allocation in primary care services in micro-regions in the Southeast of Brazil. In order to evaluate the efficiency of the health care sector, variables that represent allocation of resources (inputs) and supplied services (outputs) were selected. The measurement of efficiency was performed by the Data Envelopment Analysis, using the classical model of variable return to scale and product-oriented. The efficient frontier obtained by this analysis does not intend to provide a rigid standard of efficiency for healthcare, but a way to check how much the regions could provide with the same amount of investment. The results showed that the performance of these micro Southeast regions can be considered as moderate and that the State of São Paulo has the greatest number of efficient micro-regions. Lastly, the paper asserts about the importance of using control tools, based on results to promote policies to improve the efficiency of primary health care, which consider the diversity of the municipalities and micro-regions of the country, according to the inter-regional particularities.

Keywords: Control. Public Healthcare. Efficiency. DEA. Public Management.

Texto completo em português: http://www.apgs.ufv.br Full text in Portuguese: http://www.apgs.ufv.br

Introdução

No Brasil, desde a década de 1990, existe a preocupação por parte de pesquisadores da área de gestão, da saúde e dos chefes de governo em verificar a qualidade e a eficiência da prestação dos serviços de saúde no setor público. Nessa direção, cabe citar os trabalhos de Serapioni (1999), Meniccuci (2003), Castiel (2008) e Mendes et al. (2011). Assim, torna-se cada vez mais necessária a utilização de técnicas e métodos que possibilitem avaliações no setor, tais como método de controle das políticas públicas de saúde.

Nesse contexto, o controle tem extrema relevância, sendo um elemento fundamental para assegurar que as atividades se realizem da forma desejada e contribuam para a manutenção e a melhoria de uma posição competitiva, bem como a consecução de estratégias, planos, programas e operações em conjunto com as necessidades identificadas pelos cidadãos. Uma vez que as organizações, tanto públicas como privadas, existam em um contexto social caracterizado por fortes mudanças, incertezas e alta competitividade, o controle existirá para orientar e proporcionar a melhoria contínua frente a esse ambiente.

O controle comportamental (ou de ação), o controle de resultados e o controle social são os principais mecanismos de controle classificados por Ouchi (1979). Essa abordagem teórica de controle, proposta há mais de 30 anos, ainda é utilizada e citada em estudos mais contemporâneos, como os de Benavent e Villarmois (2006), Chtioui e Thiery-Dubuisson (2011), Albert e Bourdeau (2012), Lans e Tomei (2014). São formas distintas de controle que podem ter maior ou menor utilidade, dependendo do grau de compreensão dos processos ou do grau de mensuração dos resultados obtidos.

A questão do controle está na capacidade do gestor ou pesquisador de utilizar informações disponíveis com o propósito de mensurar o desempenho de alguma organização ou programa. 
Devido à disponibilidade de dados secundários fornecidos pelo Ministério da Saúde e à possibilidade de utilizá-los como ferramenta de controle, este estudo focará na pressuposição do controle por resultados. Apesar de organizações públicas e da saúde já utilizarem indicadores como o IDH e coeficientes epidemiológicos e de morbidade, entre outros, para o controle da situação da saúde, ainda são escassos indicadores de eficiência relativa e programação linear para comparar regiões distintas. Focando-se nesse tipo de controle, este estudo propõe-se a responder a seguinte pergunta: qual a eficiência relativa da alocação de recursos públicos na atenção básica da saúde, em microrregiões do Sudeste do Brasil, entre 2008 e 2014 ?

O foco contemporâneo em metodologias de controle no setor público está relacionado às diversas reformas que governos e analistas de políticas públicas têm introduzido na contemporaneidade, geralmente adotando mecanismos de controle associados com o setor privado (Aubert \& Boudeau, 2012).

No caso da saúde em particular, após a descentralização de suas ações e serviços com a Constituição de 1988, os recursos financeiros passaram a ser transferidos do Ministério da Saúde para os fundos municipais de saúde, ficando a administração dos recursos a cargo do gestor municipal e o controle social a cargo dos conselhos de saúde. Atualmente, grande parte das ações de saúde municipais são decididas em nível microrregional, tendo em vista que o atual processo de descentralização da saúde brasileiras promove a atuação em rede dos municípios, com um (ou mais) município(s) da microrregião como referência da rede de atenção à saúde microrregional.

O presente estudo teve como objetivo analisar a eficiência alocativa de recursos públicos na atenção primária da saúde, tomando como referência as microrregiões do Sudeste do Brasil, a partir da classificação das Secretarias de Estado da Saúde de cada estado. O estudo está pautado na pressuposição factual da necessidade de eficiência da aplicação dos recursos no setor público.

Portanto, considerando a existência limitada de recursos e o fato que ainda milhões de brasileiros não possuem acesso aos serviços básicos de saúde, é oportuno mensurar a eficiência das microrregiões do Sudeste e propor um modelo para análise e controle de resultados. A ampla gama de dados disponíveis pode ser uma alternativa de controle e monitoramento para que os próprios municípios dessas microrregiões possam buscar benchmarkings no intuito de aumentar a oferta de atendimento aos cidadãos.

Cabe ressaltar que este estudo toma como referência os trabalhos de O'Toole (1999) e Bretschneider et al. (2007), que mensuraram a eficiência em educação por meio da análise envoltória de dados (DEA), e Marinho (2001; 2003), Faria et al. (2008), Gonçalves et al. (2008), Gonçalves et al. (2012), Santos et al. (2014), que discorrem sobre o desempenho na área de saúde. As pesquisas e produções científicas citadas têm contribuído para a avaliação comparativa de eficiência na área, estabelecendo unidades de referência interna em estudos direcionados a hospitais, clínicas e serviços ambulatoriais de saúde.

No próximo tópico, algumas das características da saúde pública brasileira serão abordadas, seguidas de discussões importantes acerca dos principais conceitos sobre controle e desempenho. Por fim, analisaremos os resultados alcançados e colocaremos à disposição as principais conclusões da pesquisa.

\section{Entendendo a política de saúde no Brasil}

Embora os índices de saúde brasileiros tenham avançado nos últimos 25 anos, o Brasil ainda ostenta um desempenho mediano em relação ao seu nível de gasto. A comparação entre nível de gasto e indicadores de saúde, como mortalidade infantil e materna, coloca o Brasil em um nível médio de desempenho na América Latina e entre outros países de renda média. Muitos outros países gastam menos e atingem indicadores de saúde iguais ou superiores (World Bank, 2013).

De acordo com Fleury e Baris (2001), a responsabilidade pela prestação dos serviços de saúde é dividida entre estados e municípios, geralmente de acordo com o nível de complexidade. Desde a promulgação da Política Nacional da Atenção Básica em 2007, como regra geral, a atenção primária (ou atenção básica, como também é conhecida) é realizada pelos municípios, e a secundária e a de alta complexidade, pelos estados e governo federal (Santos et al., 2014; Gonçalves et al., 2015).

O processo de descentralização impôs a municipalização de forma radical; os municípios passaram a assumir as funções de coordenação e gestão da política de saúde local, devendo cumprir as metas dos programas nacionais, utilizando os recursos destinados pelos governos federal, estadual e municipal. A organização das ações da rede de saúde é atualmente estabelecida por um grupo de municípios das microrregiões de saúde com características epidemiológicas similares e que possibilitem a integralidade por meio da referência e contrareferência (Gonçalves et al., 2015).

Dentre as diversas estratégias que foram e continuam sendo utilizadas com a intenção de melhorar o acesso aos serviços de saúde, a maioria são de programas que buscam a provisão de serviços de cuidados primários, uma vez que estes têm sido considerados a porta de entrada aos sistemas de saúde. Além disso, os serviços de cuidados primários têm-se mostrado efetivos em reduzir as injustiças no acesso aos cuidados de saúde em diversas populações (Starfield, 2005). Dentre essas estratégias, diversos países europeus empreenderam reformas em seus sistemas de saúde, com propostas substanciais de investimento no financiamento, na organização e oferta de serviços (Rechel et al., 2009).

$\mathrm{Na}$ atenção básica, os recursos devem ser realocados para tornar as políticas da atenção básica uma realidade e para se utilisarem esses recursos com o intutito de reduzir inequidades $e$ descompassos no sistema de saúde. A atividade de alocação de recursos é tanto técnica quanto política. Deve-se considerar que a alocação de recursos está baseada na necessidade e na 
equidade, em oposição à demanda, e isso implica decisões políticas e não apenas econômicas (Segall, 1983)

Costa et al. (2011) e Mendes (2012) afirmam que o modelo de atenção hegemônico ainda se caracteriza pelo enfoque saúdedoença-cuidado, desconsiderando seus determinantes sociais, com ações desarticuladas, desintegradas, pouco cuidadoras, centradas na assistência médico-hospitalar especializada e com incorporação acrítica de novas tecnologias, constituindo-se em um modelo caro e pouco eficiente. Nesse sentido, as análises da gestão da saúde em nível microrregional tornam-se importantes, com o propósito de avaliar a eficiência das redes de atenção à saúde.

Segundo Mendes (2012), a mudança do modelo de atenção à saúde envolve a adoção de práticas integrais. Atualmente, o conjunto de recursos tecnológicos está alocado em ações desarticuladas, centradas na assistência médico-hospitalar especializada e incorporando de forma acrítica as novas tecnologias, configurando-se num modelo pouco eficiente. Há uma reflexão acerca do modelo que rege o nosso atual sistema de saúde, em busca de alternativas em prol de um atendimento integral e de qualidade.

Ações de promoção e proteção da saúde são fundamentais para a reorientação dos modelos assistenciais, sendo uma estratégia de articulação transversal que objetiva a melhoria na qualidade de vida e a redução dos riscos à saúde por meio da construção de políticas públicas saudáveis, que proporcionem melhorias no modo de viver (Czeresnia, 1999; Brasil, 2007).

Para a efetivação do SUS é necessário o fortalecimento da atenção primária, entendendo-a como o contato preferencial dos usuários com o sistema de saúde (Brasil, 2007; 2012). É fundamental, no entanto, a garantia do acesso dos usuários aos serviços de média e alta complexidade, assegurando a integralidade da atenção.

Na organização da atenção básica, um aspecto fundamental é o conhecimento do território, que não pode ser compreendido apenas como um espaço geográfico, delimitado para constituir a área de atuação dos serviços. Ao contrário, deve ser reconhecido como "Espaço Social", onde, ao longo da história, a sociedade foise constituindo e, por meio do processo social de produção, dividindo-se em classes diferenciadas, com acessos também diferenciados aos bens de consumo, incluídos os serviços de saúde (Brasil, 2006; Elias et al., 2006; Thiede \& Mcintyre, 2008).

Assim, conhecer o território implica um processo de reconhecimento e apropriação do espaço local e das relações da população da área de abrangência com a unidade de saúde, levando em consideração dados como perfil demográfico e epidemiológico da população, contexto histórico e cultural, equipamentos sociais (associações, igrejas, escolas, creches), lideranças locais e outros considerados relevantes para intervenção no processo saúde-doença. A apropriação do espaço local é fundamental, pois os profissionais de saúde e a população poderão desencadear processos de mudança das práticas de saúde, tornando-as mais adequadas aos problemas da realidade local (Brasil, 2007).
Para analisar a eficácia das ações de atenção primária no país, diversos analistas têm focado na avaliação dos indicadores de internações por condições sensíveis à atenção primária (ICSAP). As condições sensíveis à atenção primária são um conjunto de problemas de saúde para os quais a efetiva ação da atenção primária diminuiria o risco de internações. São denominadas ainda de Condições Sensíveis à Atenção Ambulatorial, do inglês Ambulatorty Care Sensitive Conditions (Alfradique et al., 2009). O conceito foi desenvolvido nos EUA no final do século $X X$, em estudos que encontraram variações das taxas de internação de condições consideradas preveníveis de acordo com a cobertura por plano de saúde ou nenhuma cobertura. Segundo Fontenelle (2011), o marco conceitual do indicador foi criado na Espanha por Caminal et al. (2004) e adaptado no Brasil por Alfradique et al. (2009), descrevendo as diferentes vias pelas quais uma pessoa pode ter acesso aos diferentes componentes do sistema de saúde, respeitando ou não o papel de primeiro contato da APS. Depois de consolidada pelo Departamento de Atenção Básica do Ministério da Saúde, a lista foi submetida a uma consulta à Sociedade Brasileira de Medicina de Família e Comunidade e a uma consulta pública, sendo enfim publicada na forma da Portaria SAS n. 211, de 17 de abril de 2008.

Segundo Alfradique et al. (2009), as ICSAP de 2000 a 2006 caíram 15,8\% no país, e o declínio dessas hospitalizações foi maior do que as internações por condições não sensíveis à atenção primária. Por outro lado, Henrique e Calvo (2008) observaram que no período de 2001 a 2004 os municípios de Santa Catarina mantiveram as mesmas taxas de ICSAP, apesar da expansão da Saúde da Família. Em 2004, 78,4\% dos municípios tinham uma cobertura por Saúde da Família considerada alta (maior que $70 \%$ ), mas $81,6 \%$ tinham fracos indicadores de mudança no modelo assistencial, um indicador composto pelos autores a partir de taxa de visitas domiciliares, taxa de exames de patologia clínica, taxa de encaminhamento a especialistas e taxa de consultas de enfermagem.

Segundo Cardoso et al. (2013), o destaque à atenção primária como foco prioritário da organização do cuidado em saúde é uma tendência tanto brasileira quanto mundial. Inicialmente planejada a partir de uma perspectiva restrita como forma de diminuir o gasto hospitalar, a atenção primária em saúde vem sendo retomada de forma mais ampla, com o intuito de desviar o foco da assistência médica e valorizar a integralidade e a longitudinalidade do cuidado em saúde como principal estratégia, em detrimento do enfoque baseado na atividade hospitalar e do enfoque curativo, fragmentado, ineficiente e pouco resolutivo. Estudos demonstram a efetividade da atenção primária, evidenciando seu alto potencial de resolutividade.

As problemáticas que envolvem o setor saúde, no Brasil, são muitas e destacam-se ao analisarmos os estabelecimentos médicos, as políticas setoriais e o modelo de saúde que vêm sendo adotado nas últimas décadas. Torna-se necessário, então, racionalizar as ações nesse âmbito, analisando as relações custoefetividade e minimizando os equívocos no direcionamento dos 
investimentos e na condução das políticas públicas voltadas ao setor, buscando sempre a otimização da aplicação dos recursos (Forbes \& Lynn, 2007).

\section{Eficiência por meio do controle e desempenho}

Segundo Gomes e Salas (2001) e Lans e Tomei (2014), o controle é um elemento fundamental para assegurar que as atividades se realizem da forma desejada e contribuam para a manutenção e melhoria da posição competitiva e a consecução das estratégias, planos, programas e operações em conjunto com as necessidades identificadas. Desse modo, as organizações existem em um contexto social caracterizado por fortes mudanças, incertezas e alta competitividade, e o controle existe para orientar e proporcionar a melhoria contínua frente a esse ambiente.

Já o controle de gestão refere-se ao processo de interrelação de um conjunto de elementos internos (formais elementos do processo de estratégia e, mais especificamente, representa um dos mecanismos administrativos que contribuem para a implementação de estratégias (Govindarajan, 1988; Mintzberg et al., 2006). Pode ser entendido como o processo de guiar as organizações em direção aos padrões viáveis de atividade em um ambiente incerto, exercendo o papel organizacional de permitir que gestores influenciem o comportamento de outros membros organizacionais na direção de estratégias adotadas (Berry, Broadbent \& Otley, 2005; Anthony \& Govindarajan, 2002).

Hatch (1997), por sua vez, considera que o controle pode ser aplicado tanto no indivíduo quanto nas unidades organizacionais e nas próprias organizações. $O$ autor compreende que, na moderna teoria organizacional, o controle é visto como mecanismo de implementação estratégica, pressupondo que a organização deve regular e certificar-se de que interesses divergentes não interfiram nas metas e estratégias da organização. Tal abordagem foca em mecanismos de controle comportamental, visando garantir que interesses individuais sejam minimizados.

Os vários mecanismos de controle disponíveis são classificados por Ouchi (1979) em: controle comportamental ou de ação; controle de resultados ou de saídas (outputs); e controle cultural ou social.

Controles comportamentais ou de ação envolvem a observação das ações dos indivíduos quando eles estão realizando suas atividades, ou seja, ocorre quando uma pessoa assume a responsabilidade pelo trabalho de outras, dando-lhes instruções e monitorando suas ações (Mintzberg, 1979). Na visão de Drury (2004), esse tipo de controle é adequado quando os relacionamentos de causa e efeito são bem entendidos, de tal forma que, se os meios apropriados são seguidos, os resultados esperados ocorrerão.

Segundo Ouchi (1979), controles culturais e sociais permitem o monitoramento mútuo entre os diversos atores organizacionais por meio de normas e valores de grupo. Esse tipo de controle é adequado quando nem os relacionamentos de causa e efeito são bem entendidos, nem os resultados são facilmente mensuráveis.
Por fim, controles de resultados ou de saídas (outputs) envolvem a coleta e a emissão de relatórios com informações relativas aos resultados do esforço de trabalho, sendo composto dos seguintes estágios: i) estabelecer medidas de desempenho que minimizem comportamento indesejável; ii) estabelecer metas de desempenho; iii) mensurar desempenho; e iv) fornecer recompensas e punições. Segundo Ouchi (1979), esse tipo de controle é adequado quando os resultados são facilmente mensuráveis, podendo as relações de causa e efeito ser ou não bem entendidas. Rosen e Baroudi (1992) entendem que primeiramente o controle adquire uma característica simplificada baseada nos resultados e, posteriormente, procura tornar-se hegemônico e mais direto por meio da institucionalização de padronizações e regras, como um mecanismo direcionado ao controle comportamental.

Entretanto, um dos grandes dilemas das organizações está justamente no terceiro item do parágrafo anterior, que consiste em saber como mensurar o desempenho de forma a conseguir eficácia global da instituição a longo prazo. Sabe-se, entretanto, que a perspicácia está localizada nos investimentos realizados em equipamentos, condições de trabalho mais adequadas e, principalmente, em treinamentos profissionais, de forma que tenha funcionários mais preparados e motivados. Esse é o caminho para o alcance do desempenho organizacional (Scott \& Davis, 2007; Selden \& Sowa, 2004).

As avaliações do desempenho organizacional são algumas vezes mensuradas por meio da medida de avaliação de eficiência e eficácia. Em relação a isso, Zeydan e Çolpan (2009) argumentam que:

Eficácia é uma medida da obtenção de resultados desejados, tais como o produto certo com a qualidade esperada. A eficiência é definida como a proporção de saída para entrada de dados. Em outras palavras, a eficácia é fazer as coisas certas, e eficiência é fazer certo as coisas. Assim, a medida de eficácia é um parâmetro estratégico que deve ser tomado em consideração ao longo prazo, a tomada de decisão, enquanto medida de eficiência é um parâmetro operacional de curto prazo de tomada de decisão.

Atualmente, em função da necessidade de maior controle gerencial e obtenção de resultados mais eficazes, as organizações buscam a melhoria de suas atividades e nesse sentido concentram esforços na busca constante de seu aprimoramento, não somente em termos de inovações tecnológicas, mas também na identificação de aspectos que podem ainda ser melhorados em termos de desempenho, a fim de se obter uma alavancagem nos negócios da empresa e facilitar o processo de tomada de decisão.

Nesse processo de melhoria contínua do desempenho é que apontam os critérios de mensuração e avaliação da eficiência que serão discutidos neste trabalho como forma de obter aumento do provimento da oferta de serviços aos cidadãos e sugerir um mecanismo de controle para o setor saúde da região Sudeste como informação para analisar a posição da maioria dos municípios ou microrregiões frente à fronteira eficiente de produção da saúde. 


\section{Métodos e procedimentos analíticos}

Este estudo tomou as microrregiões de saúde dos quatro estados do Sudeste como referência para a análise da eficiência da saúde. O principal instrumento utilizado na coleta de dados foi a pesquisa documental, por meio de relatórios e estatísticas disponíveis pelo Ministério da Saúde.

A Constituição Federal de 1988 define que microrregião constitui um agrupamento de municípios limítrofes, que têm como meta a integração, organização, planejamento e execução de funções públicas de interesse comum, definidas com referências políticas, econômicas e sociais. No setor saúde, as microrregiões são definidas de acordo com o Plano Diretor de Regionalização de cada estado e têm o propósito de definir estratégias diferentes para regiões diferentes, de acordo com a característica epidemiológica de cada região.

A análise da eficiência utilizou o modelo de Análise Envoltória de Dados, por meio de uma relação de produtividade que compara a relação entre resultados produzidos em saúde sobre insumos alocados. Em outros termos, foram selecionadas variáveis que representaram os recursos (inputs) e outras variáveis que representaram os serviços ou produtos oferecidos à sociedade (outputs). A mensuração da eficiência foi realizada por intermédio da Análise Envoltória de Dados (DEA), com a utilização do modelo clássico de retornos variáveis com orientação para o produto (Charnes et al., 1978; Banker et al., 1984; Charnes et al., 1994; Belloni, 2000; Estelita Lins \& Meza, 2000).

A definição das variáveis inseridas na análise de eficiência da saúde do Sudeste considerou a estratégia mais recente do Ministério da Saúde que busca inserir a atenção primária como núcleo da rede de atenção à saúde do país, conforme publicado na Política Nacional da Atenção Básica de 2007 e sua atualização em 2011. Então, foram selecionadas variáveis anuais de 2008 a 2014, dos indicadores do rol de Diretrizes, Objetivos e Indicadores 2014-2015, estabelecidos na Resolução n. 5 da Comissão Intergestores Tripartite, de 19 de junho de 2013. As variáveis foram:

\section{Recursos/Insumos - inputs $(\mathrm{X})$ :}

- Cobertura da atenção básica: pertence ao objetivo 1.1 das diretrizes da Resolução n. 5, de 19 de junho de 2013;utilização de mecanismos que propiciem a ampliação do acesso a atenção básica. É a cobertura populacional estimada pelas equipes de atenção básica atualizados a partir do Cadastro Nacional de Estabelecimentos de Saúde (CNES).

- Cobertura de acompanhamento das condicionalidades de saúde do Programa Bolsa Família: pertencente ao mesmo objetivo 1.1, refere-se ao número de famílias beneficiárias do Programa Bolsa Família com perfil saúde acompanhadas pela atenção básica.

- Cobertura populacional estimada pelas equipes básicas de saúde bucal: estimativa da população coberta pelas equipes de saúde bucal, atualizados a partir do CNES. Também pertencente ao objetivo 1.1.

- Número de Equipes de Saúde per capita: número de equipes de saúde per capita, segundo CNES.

Produtos/Serviços - outputs (Y):

- Inverso da proporção de internações por condições sensíveis à atenção primária (ICSAB): refere-se ao inverso da proporção de internações por causas selecionadas sensíveis à atenção básica, por local de residência e ano de internação. Também faz parte do objetivo 1.1.

- Inverso da taxa de mortalidade infantil: inverso da proporção de óbitos em menores de 1 ano de idade em um determinando local de residência e ano. Faz parte do objetivo 3.2, que é organizar a rede de atenção à saúde materna e infantil para garantir acesso, acolhimento e resolutividade.

As variáveis inseridas são importantes para representar o atual esforço do Ministério da Saúde na reorganização das redes de atenção em saúde, tomando como referência a atenção básica. A cobertura populacional da atenção básica, da saúde bucal e da população beneficiada pelo Bolsa Família explicam teoricamente o esforço colocado pelo estado na alocação de recursos na saúde das microrregiões analisadas.

A cobertura e o número de equipes per capita foram colocados concomitantemente, porque a cobertura pode variar em municípios com o mesmo número de equipes, uma vez que a cobertura é influenciada por fatores demográficos e socioeconômicos da região. A atenção básica é caracterizada como um nível de atenção com baixa densidade tecnológica e alta complexidade, fazendo com que a atuação das equipes seja primordial para o sucesso das ações e serviços de saúde. Então, foi inserido o número de equipes de saúde per capita como insumo para o modelo de eficiência que complementa a informação das coberturas. Todas estas indicam os principais esforços de alocação de recursos públicos na saúde, considerando o nível de atenção básica.

As variáveis produto do modelo foram o inverso da ICSAP e da mortalidade infantil, tendo em vista que a alocação de recursos na saúde pública tem o objetivo de reduzir, pelo menos, esses dois índices. Apesar do indicador ICSAP ser um indicador mais recente na avaliação da saúde, a mortalidade infantil é um indicador tradicional, utilizado nas avaliações de saúde da Organização Mundial da Saúde, do Banco Mundial, Nações Unidas e Ministério da Saúde.

No intuito de incorporar a possibilidade de retornos variáveis à escala, Banker et al. (1984) propuseram o modelo conhecido como BCC, da análise envoltória de dados, apresentado no Problema de Programação Linear da Equação 1. 
$\operatorname{Max} \theta^{\mathrm{RVE}}$

Sujeito a :

$\sum_{j=1}^{n} \lambda_{j} x_{i j} \leq x_{i 0,}, i=1,2, \ldots, m$

$\sum_{j=1}^{n} \lambda_{j} y_{r j} \geq \theta^{R V E} y_{i 0}, r=1,2, \ldots, s$

$\sum_{j=1}^{n} \lambda_{j}=1$

$\sum_{\mathrm{j}=1}^{\mathrm{n}} \lambda_{\mathrm{j}} \geq 0, \forall \mathrm{j}$

Equação 1

considerando que as microrregiões de saúde utilizem $m$ insumos e $s$ produtos, $j$ está associado a microrregião 0 analisada e $n$ corresponde ao número total de DMUs; $x_{i j}$ é a quantidade consumida do insumo $i(i=1,2 \ldots, m)$ pela microrregião j; $y_{r j}$ é a quantidade produzida do produto $r(r=1,2,3 . . s)$ pela microrregião j; $\lambda_{j}$ é o peso da microrregião j na composição da microrregião 0 virtual analisada (também contendida como projeção na fronteira eficiente).

Para maiores esclarecimentos sobre a modelagem utilizada sugere-se a leitura de Charnes et al. (1978), Charnes et al. (1994), Banker et al. (1984) e Surco (2004).

\section{Análise dos resultados}

As análises foram realizadas com as variáveis do nível de atenção básica, que no Brasil caracterizam-se principalmente por prevenção, promoção, consultas e exames de baixa densidade tecnológica. Essa explanação é importante, pois, quanto maior o número dos procedimentos diagnosticados à saúde, mais complexo se torna o controle, dado o nível de incerteza das atividades. Os procedimentos mais complexos tendem a possuir mais peculiaridades, e o controle por resultados poderia não ser suficiente para tirar conclusões (ver Ouchi, 1979). Isso significa dizer que, geralmente, os processos da atenção primária buscam resolver a maioria dos problemas de saúde antes das complicações, reduzindo o nível de incerteza de suas atividades.

Outro ponto importante a ser explicado é que a construção de uma fronteira de eficiência para comparar as microrregiões não tem a intenção de construir um parâmetro para o setor de saúde, mas incorporar um instrumento adicional de controle de resultados. Assim, o que se pretende é comparar as microrregiões para indicar o que é investido e produzido, sendo que o mais eficiente no grupo pode estar sendo ineficiente sob a visão de outras perspectivas da saúde e vice-versa. Assim, as microrregiões que estão sobre a fronteira de eficiência (Charnes et al., 1978; Banker et al., 1984; Charnes et al., 1994) não são consideradas meta para o setor de saúde, mas, sim, uma forma de controle, dado o contexto proposto neste trabalho e somente para a microrregião analisada.

A Tabela 1 exibe as estatísticas descritivas das variáveis utilizadas no modelo de eficiência e dos escores de eficiência entre os anos 2008 e 2014. A média geral para as 153 microrregiões, durante os 7 anos, mostrou uma eficiência de, aproximadamente, $72 \%$. Ademais, a Tabela 1 permite inferir que existem discrepâncias entre a eficiência das microrregiões, pois foram apresentadas pelo modelo microrregiões com um índice de eficiência que variam de 38 a $100 \%$ quando se analisa apenas a variação ao longo dos anos, sem considerar as diferenças entre os municípios. Ao longo dos 7 anos analisados, observou-se que em média, os municípios variam a eficiência, aproximadamente, de 40 até $100 \%$. Essa variação pode estar relacionada com o período de mudanças no sistema de saúde implementadas após a Política Nacional da Atenção Básica e Pactos pela Saúde, que exigiram uma maior municipalização da saúde, principalmente, via atenção primária.

Tabela 1

\begin{tabular}{|c|c|c|c|c|c|c|}
\hline \multicolumn{7}{|c|}{ Variáveis e escores de eficiência das microrregiões de saúde do Sudeste } \\
\hline Variáveis & & Média & Desvio & Mínimo & Máximo & Observações \\
\hline \multirow{4}{*}{ Cobertura da atenção básica } & Total & 74,44 & 16,23 & 27,23 & 100 & $N=918$ \\
\hline & Entre municípios & & 15,27 & 37,07 & 100 & $n=153$ \\
\hline & Entre anos & & 5,61 & 48,40 & 107,91 & $\mathrm{~T}=6$ \\
\hline & Total & 56,04 & 26,14 & 0,10 & 100,00 & $N=918$ \\
\hline \multirow[t]{3}{*}{ Cobertura da saúde bucal } & Entre municípios & & 17,97 & 14,38 & 100,00 & $\mathrm{n}=153$ \\
\hline & Entre anos & & 19,03 & $-26,48$ & 88,70 & $\mathrm{~T}=6$ \\
\hline & Total & 0,40 & 1,79 & 0,004 & 24,50 & $N=1071$ \\
\hline \multirow{2}{*}{$\begin{array}{l}\text { Equipes de saúde per capita } \\
\text { (1000 habitantes) }\end{array}$} & Entre municípios & & 1,79 & 0,006 & 22,21 & $n=153$ \\
\hline & Entre anos & & 0,24 & $-6,09$ & 2,69 & $\mathrm{~T}=7$ \\
\hline \multirow{4}{*}{$\begin{array}{l}\text { Cobertura de beneficiários do } \\
\text { bolsa família }\end{array}$} & Total & 70,11 & 14,05 & 21,20 & 96,76 & $N=918$ \\
\hline & Entre municípios & & 10,62 & 39,35 & 90,06 & $n=153$ \\
\hline & Entre anos & & 9,23 & 32,05 & 97,46 & $\mathrm{~T}=6$ \\
\hline & Total & 36,20 & 15,71 & 17,34 & 95,86 & $N=918$ \\
\hline \multirow[t]{3}{*}{ ICSAP } & Entre municípios & & 9,91 & 18,22 & 64,67 & $n=153$ \\
\hline & Entre anos & & 12,21 & 21,19 & 92,57 & $\mathrm{~T}=6$ \\
\hline & Total & 13,50 & 3,71 & 3,65 & 35,50 & $N=918$ \\
\hline \multirow[t]{3}{*}{ Mortalidade infantil } & Entre municípios & & 2,84 & 9,12 & 24,96 & $n=153$ \\
\hline & Entre anos & & 2,39 & 1,33 & 25,82 & $\mathrm{~T}=6$ \\
\hline & Total & 72,38 & 17,49 & 30,59 & 100,00 & $N=1071$ \\
\hline \multirow[t]{2}{*}{ Escore de eficiência } & Entre municípios & & 15,32 & 37,98 & 100,00 & $\mathrm{n}=153$ \\
\hline & Entre anos & & 8,50 & 40,15 & 100,00 & $\mathrm{~T}=7$ \\
\hline
\end{tabular}

Fonte: resultado da pesquisa. 
A Tabela 1 também mostra que, em média, as microrregiões possuíram nos últimas 7 anos uma cobertura de $77,44 \%$ na atenção básica, 56,04\% na saúde bucal e 70\% dos beneficiários do Bolsa Família. Isso evidencia que as regiões ainda passam por um período de transição, tendo em vista que o objetivo da atenção básica é dar cobertura para $100 \%$ da população. Além disso, as microrregiões apresentam em média 0,4 equipes por mil habitantes, 36\% de ICSAP e um índice de mortalidade infantil de 13,5 .
O Gráfico 1 permite uma melhor visualização das diferenças entre os escores de eficiência das microrregiões em cada ano. É possível inferir que existe uma diferença no nível de ICSAP e mortalidade infantil, mantidas constantes as coberturas da atenção básica, da saúde bucal, dos beneficiários do Bolsa Família e do número de equipes de saúde per capita.

Gráfico 1 - Eficiência das microrregiões do Sudeste entre 2008 e 2014.
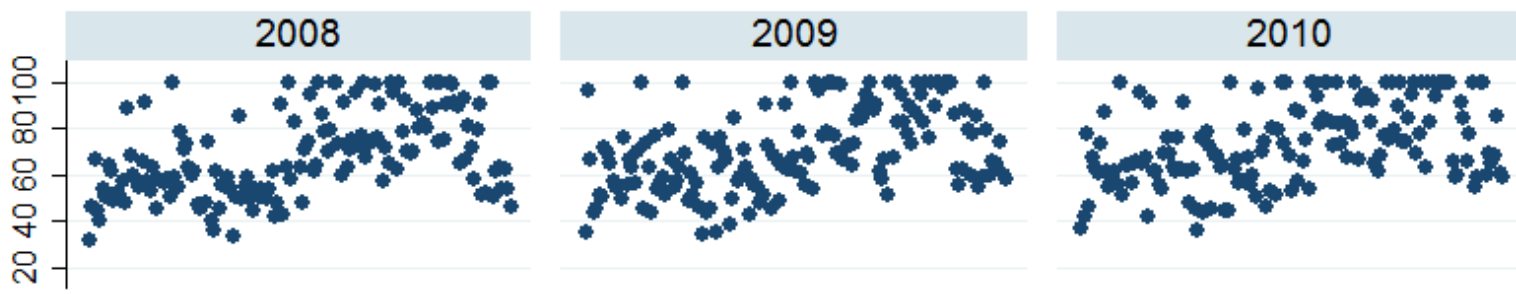

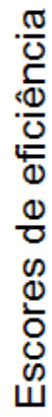
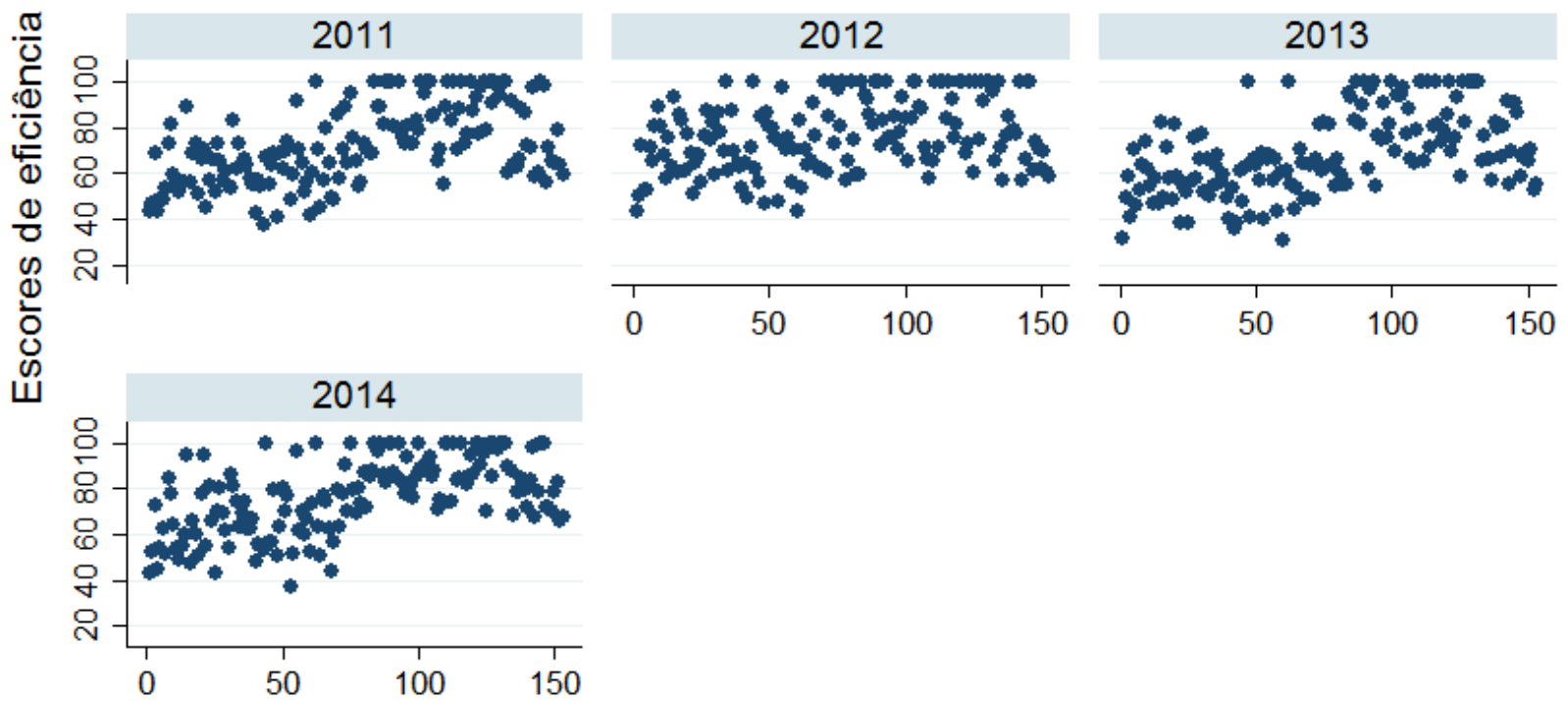

Gráficos por ano

\section{Microrregiões de saúde}

Fonte: elaboração própria

O Gráfico 1 mostra que existem microrregiões na fronteira que estão identificadas na Tabela 2. No Estado de Minas Gerais, apenas três microrregiões permaneceram eficientes por mais de um ano. As microrregiões de Montes Claros/Bocaiúva, Santos Dumont, Uberlândia/Araguari e Varginha foram as que mais permaneceram na fronteira.
No Rio de Janeiro, apenas a microrregião Metropolitana I e II apresentaram eficientes em todos os anos. O estado de São Paulo apresentou o maior número de microrregiões eficientes. Considerando os 7 anos, 47 microrregiões foram eficientes, sendo 31 somente no estado de São Paulo. 
Tabela 2

Microrregiões eficientes do Sudeste brasileiro

\begin{tabular}{|c|c|c|c|c|c|c|c|c|c|c|c|c|c|c|}
\hline Ano & UF & Microrregião & Ano & UF & Microrregião & Ano & UF & Microrregião & Ano & UF & Microrregião & Ano & UF & Microrregião \\
\hline 2010 & MG & Contagem & 2012 & RJ & Metropolitana II & 2013 & SP & Campinas & 2010 & SP & Mananciais & 2012 & SP & Rota dos Bandeirantes \\
\hline 2009 & MG & Formiga & 2014 & RJ & Metropolitana II & 2014 & SP & Campinas & 2011 & SP & Mananciais & 2013 & SP & Rota dos Bandeirantes \\
\hline 2008 & MG & Itaúna & 2012 & SP & Alta Mogiana & 2008 & SP & Coração do DRS III & 2012 & SP & Mananciais & 2014 & SP & Rota dos Bandeirantes \\
\hline 2012 & MG & Januária & 2008 & SP & Alto do Tietê & 2010 & SP & Coração do DRS III & 2013 & SP & Mananciais & 2009 & SP & Santa Fé do Sul \\
\hline 2009 & MG & João Pinheiro & 2009 & SP & Alto do Tietê & 2013 & SP & Coração do DRS III & 2014 & SP & Mananciais & 2013 & SP & Santa Fé do Sul \\
\hline 2012 & MG & Montes Claros Bocaiúva & 2010 & SP & Alto do Tietê & 2014 & SP & Coração do DRS III & 2011 & SP & Norte - Barretos & 2010 & SP & São José do Rio Preto \\
\hline 2014 & MG & Montes Claros Bocaiúva & 2011 & SP & Alto do Tietê & 2011 & SP & Extremo Oeste Paulista & 2012 & SP & Norte do DRS III & 2012 & SP & São José do Rio Preto \\
\hline 2013 & MG & Ouro Preto & 2012 & $\mathrm{SP}$ & Alto do Tietê & 2011 & SP & Franco da Rocha & 2009 & SP & Oeste VII & 2013 & SP & São José do Rio Preto \\
\hline 2010 & MG & Poços de Caldas & 2014 & SP & Alto do Tietê & 2012 & SP & Franco da Rocha & 2010 & SP & Oeste VII & 2014 & SP & São José do Rio Preto \\
\hline 2011 & MG & Santos Dumont & 2010 & SP & Alto Vale do Paraíba & 2009 & SP & Grande ABC & 2012 & SP & Oeste VII & 2008 & SP & São Paulo \\
\hline 2014 & MG & Santos Dumont & 2013 & SP & Araras & 2012 & SP & Grande ABC & 2014 & SP & Oeste VII & 2010 & SP & São Paulo \\
\hline 2012 & MG & Três Pontas & 2011 & SP & Assis & 2008 & SP & José Bonifácio & 2014 & SP & Ourinhos & 2011 & SP & São Paulo \\
\hline 2008 & MG & Uberlândia Araguari & 2008 & SP & Baixa Mogiana & 2013 & SP & José Bonifácio & 2008 & SP & Piracicaba & 2012 & SP & São Paulo \\
\hline 2010 & MG & Uberlândia Araguari & 2009 & SP & Baixa Mogiana & 2014 & SP & José Bonifácio & 2009 & SP & Piracicaba & 2013 & SP & São Paulo \\
\hline 2012 & MG & Uberlândia Araguari & 2010 & SP & Baixa Mogiana & 2009 & SP & Jundiaí & 2010 & SP & Piracicaba & 2014 & SP & São Paulo \\
\hline 2009 & MG & Varginha & 2011 & SP & Baixa Mogiana & 2010 & SP & Jundiaí & 2011 & SP & Piracicaba & 2009 & SP & Sorocaba \\
\hline 2010 & MG & Varginha & 2012 & SP & Baixa Mogiana & 2011 & SP & Jundiaí & 2012 & SP & Piracicaba & 2010 & $\mathrm{SP}$ & Sorocaba \\
\hline 2012 & MG & Viçosa & 2014 & SP & Baixa Mogiana & 2012 & SP & Jundiaí & 2008 & SP & Rio Claro & 2011 & SP & Sorocaba \\
\hline 2010 & RJ & Baixada Litorânea & 2008 & SP & Baixada Santista & 2013 & SP & Jundiaí & 2009 & SP & Rio Claro & 2013 & SP & Sorocaba \\
\hline 2012 & $\mathrm{RJ}$ & Baixada Litorânea & 2009 & SP & Baixada Santista & 2014 & SP & Jundiaí & 2010 & SP & Rio Claro & 2014 & SP & Sorocaba \\
\hline 2010 & $\mathrm{RJ}$ & Metropolitana I & 2012 & SP & Baixada Santista & 2010 & $\mathrm{SP}$ & Limeira & 2013 & SP & Rio Claro & 2010 & $\mathrm{SP}$ & Três Colinas \\
\hline 2011 & $\mathrm{RJ}$ & Metropolitana I & 2013 & SP & Baixada Santista & 2011 & SP & Limeira & 2014 & SP & Rio Claro & 2012 & SP & Três Colinas \\
\hline 2012 & $\mathrm{RJ}$ & Metropolitana I & 2014 & SP & Baixada Santista & 2012 & $\mathrm{SP}$ & Limeira & 2008 & SP & Rota dos Bandeirantes & & & \\
\hline 2014 & $\mathrm{RJ}$ & Metropolitana I & 2010 & SP & Campinas & 2013 & SP & Limeira & 2009 & SP & Rota dos Bandeirantes & & & \\
\hline 2008 & RJ & Metropolitana II & 2011 & SP & Campinas & 2014 & SP & Limeira & 2010 & SP & Rota dos Bandeirantes & & & \\
\hline 2010 & RJ & Metropolitana II & 2012 & SP & Campinas & 2012 & SP & Lins & 2011 & SP & Rota dos Bandeirantes & & & \\
\hline
\end{tabular}

Fonte: resultados da pesquisa 
Analisando a Tabela 3, é possível perceber grande amplitude da variação dos escores de eficiência, sendo maior no período de 2013, indo de 30,58 a $100 \%$, com uma média de aproximadamente $66,19 \%$. Portanto, faz-se necessária uma investigação mais apropriada das condições de distribuição e concentração dos dados. Esses escores de eficiência mostram o quanto as microrregiões estão produzindo em relação às microrregiões mais eficientes.

Tabela 3

\begin{tabular}{|c|c|c|c|c|c|c|c|}
\hline Ano & Média & $25^{\circ}$ & $50^{\circ}$ & $75^{\circ}$ & Mínimo & & Desvio \\
\hline 2008 & 63,32 & 53,81 & 63,32 & 80,83 & 32,27 & 100 & 18,01 \\
\hline 2009 & 70,70 & 57,50 & 67,66 & 84,56 & 34,70 & 100 & 17,83 \\
\hline 2010 & 73,52 & 61,37 & 69,19 & 88,15 & 36,16 & 100 & 17,44 \\
\hline 2011 & 72,26 & 57,88 & 70,45 & 86,88 & 37,50 & 100 & 17,15 \\
\hline 2012 & 76,80 & 64,63 & 75,35 & 89,20 & 43,49 & 100 & 15,69 \\
\hline 2013 & 66,19 & 55,50 & 66,19 & 81,49 & 30,58 & 100 & 18,03 \\
\hline 2014 & 76,48 & 63,43 & 77,96 & 88,34 & 36,88 & 100 & 16,40 \\
\hline
\end{tabular}

As fronteiras de eficiência exibidas no Gráfico 1 não buscam fornecer um padrão rígido de eficiência para a saúde, como discutido anteriormente. Essa fronteira de eficiência é uma forma de utilizar os resultados produzidos pelo setor de atendimento primário de saúde para verificar o quanto as microrregiões poderiam produzir mantendo-se constante a quantidade de investimentos. Considera-se que os processos do atendimento primário são mais plausíveis de quantificação dada sua ação na busca pela redução de incertezas. Assim, uma comparação entre microrregiões poderia ser importante para discutir e pensar sobre os motivos pelos quais algumas regiões conseguem com o mesmo esforço, obter melhores resultados. Essa é uma vantagem do modelo DEA, que compara as microrregiões em condições proporcionais de alocação de recursos.

A Tabela 3 mostrou um crescimento das medianas $\left(50^{\circ}\right.$ percentil), com exceção do ano de 2013. Visualmente, é possível observar no Gráfico 1 que um grupo de municípios se distancia mais da fronteira eficiente, sendo a explicação mais plausível para redução brusca da média e mediana. Esse fato pode estar associado tanto à melhoria da produtividade das microrregiões eficientes quanto à piora da produtividade dos municípios que já eram ineficientes. Este estudo não permite concluir o que levou a decaída de eficiência no ano de 2013.

Para a avaliação qualitativa dos resultados, foi construído um critério de categorização das DMUs em razão dos resultados, tomando-se como referência a média e o desvio-padrão, enquanto principais estatísticas descritivas apuradas. Pela Tabela 4, podemos verificar que aproximadamente $17 \%$ das microrregiões obtiveram um desempenho "baixo", considerado inferior à média mais um desvio padrão. Em um nível "médio" ficaram aproximadamente $62 \%$ da amostra, entre mais ou menos um desvio padrão ao redor da média. Com desempenho relativo superior ficaram aproximadamente $21 \%$ da amostra acima da média mais um desvio padrão.
Tabela 4

Classificação de desempenho das microrregiões, conforme escore de eficiência da abordagem DEA.

\begin{tabular}{|c|c|c|c|}
\hline Critério & Escores & Desempenho & $\begin{array}{l}\% \text { das } \\
\text { DMUs }\end{array}$ \\
\hline $\begin{array}{l}\text { Inferior a Média } \\
\text { menos } 1 \text { Desvio- } \\
\text { Padrão }\end{array}$ & $E<54,88$ & Baixo & $16,85 \%$ \\
\hline $\begin{array}{l}\text { Média mais ou } \\
\text { menos } 1 \text { Desvio- } \\
\text { Padrão }\end{array}$ & $\begin{array}{l}54,88<E \\
<89,88\end{array}$ & Médio & $62,15 \%$ \\
\hline $\begin{array}{l}\text { Superior a Média } \\
\text { menos } 1 \text { Desvio- } \\
\text { Padrão }\end{array}$ & $E>89,88$ & Alto & $21,00 \%$ \\
\hline
\end{tabular}

Fonte: Resultado da pesquisa

Vale destacar que 47 microrregiões apresentaram escores máximos, representando, portanto, benchmarks para políticas públicas no setor, quando analisada a atenção primária da saúde. Foi encontrado destaque para as microrregiões do estado de São Paulo, que, além de possuir o maior número de eficientes, também apresentou microrregiões na fronteira em mais de dois anos consecutivos. Por outro lado, nenhuma das microrregiões do Espírito Santo foi considerada eficiente.

Como sugerem Rosen e Baroudi (1992), para que essas microrregiões possam realmente ser consideradas benchmarks, o controle mais simplificado, baseado nos resultados (saídas), deve ser estendido para uma análise mais qualitativa (controle comportamental), próxima dessas microrregiões, investigando quais são os processos utilizados por estas para que elas possam atender mais cidadãos e famílias (cobertura), dado o mesmo nível de recursos utilizados pelas outras microrregiões, e com isso buscar redução nas ICSAP e mortalidade infantil. O controle por resultados (Ouchi, 1979, Anthony, 1965) seria a primeira etapa para que posteriormente fosse executasse a ação in loco nas microrregiões ineficientes identificadas na análise. Assim, a análise do controle pela eficiência relativa torna-se uma ferramenta gerencial interessante para a administração pública, tendo como foco algumas microrregiões em vez de realizar uma análise simultânea em todas as microrregiões. Como algumas das ações de políticas de saúde são descentralizadas, ao comando dos municípios, torna-se relevante a comparação entre os mais eficientes e menos eficientes. Os responsáveis pelas políticas de saúde dos municípios pertencentes as regiões menos eficientes devem utilizar-se do benchmarking para compreender como são os processos dos municípios pertencentes as microrregiões mais eficientes.

Gimzauskiene e Valanciene (2010) citam que hoje em dia as organizações devem estar alertas para a gestão eficaz dos processos, o desenvolvimento contínuo, a atualização de novas tecnologias e o gerenciamento de seus recursos humanos, além de serem flexíveis às mudanças do ambiente.

De acordo com o conceito de eficiência de Zeydan e Çolpan (2009), pode-se perceber pelas análises que algumas microrregiões poderiam ser mais eficientes, produzindo mais com os recursos que possuem, já que algumas microrregiões apresentam melhores resultados com o atual nível de input. 
Simplificando conceitos, essas microrregiões poderiam realizar mais atendimentos, mais exames e acompanhar mais famílias. Entretanto, este estudo não entrará no mérito de explicar por que algumas microrregiões produzem menos do que outras, pois, nesse caso, deveriam ser analisados os processos de maneira mais analítica e in loco, o que, por sua vez, poderia ser uma extensão deste estudo. Daí, mais uma vez, a importância fundamental do controle para a administração pública, que, segundo Anthony (1965), consiste em "garantir que as organizações utilizem seus recursos efetivamente e eficientemente atingindo seus objetivos".

Uma das possíveis razões para a disparidade dos resultados pode ter motivação na descentralização dos serviços de saúde, na qual os municípios possuem maior poder de decisão para estabelecer sua política de saúde (Marinho, 2001). Então, essa é mais uma justificativa para um controle de resultados como o proposto neste trabalho.

Esses resultados visam mostrar que a ferramenta de programação linear como ferramenta de controle pode ser o uma das ações iniciais para garantir que a organização pública utilize seus recursos eficientemente atingindo seus objetivos, conforme proposto por Gomes e Salas (2001), Anthony (1965), Emmanuel, Otley e Merchant (1993). Como citado por esses autores, o controle gerencial, aqui aplicado na administração pública, pode ser visto como um dos elementos do processo de estratégia, ou seja, elaboração de políticas públicas (Govindarajan, 1988; Mintzberg et al., 2006).

\section{Considerações finais}

Os resultados demonstraram que, no contexto geral, o desempenho das microrregiões do Sudeste na otimização de recursos alocados em atenção primária pode ser considerado mediano. Apesar disso, houve aumento da média e mediana da eficiência das microrregiões ao longo dos anos de 2008 a 2014, indicando que estas têm ganhado produtividade na alocação de recursos na atenção primária. O estado de São Paulo apresentou o maior número de microrregiões eficientes e, por outro, o Espírito Santo não apresentou nenhuma.

Em resumo, as principais contribuições deste estudo para o conhecimento estão na proposição de um modelo de controle por resultados que considera a estratégia de reposicionamento das ações e serviços de saúde do Ministério da Saúde por meio da atenção básica e a identificação e identificação de uma fronteira eficiente que pode ser utilizada como benchmarking para outros municípios e microrregiões do Sudeste. Preocupa o fato da alta disparidade regional, uma vez que o gráfico de dispersão aponta para disparidades na gestão da saúde. Apesar de baseado apenas nos resultados, esse controle pode ser utilizado como parâmetro para inserções de controle comportamental e de processos, no intuito de que resultados mais detalhados sejam obtidos em análises futuras.

Os resultados apontam para lacunas gerenciais capazes de serem supridas por meio de políticas públicas com intervenções qualitativas e quantitativas, que podem ser executadas através de investigações in loco, tomando como referência os resultados apresentados neste trabalho, juntamente com os outros estudos nele discorridos.

Os resultados apontam também as microrregiões que servem de benchmarkings para as demais, o que depõe a favor da maior troca de experiências entre os gestores inter-regionais, fato que pode ser estimulado por meio das redes de relacionamento institucionais, fomentadas, em parte, pelas secretarias regionais e consórcios intermunicipais de saúde ou por meio de uma política estadual que tenha como objetivo melhorar a eficiência dos serviços na área de saúde.

Entre as principais limitações deste estudo está o fato de que o controle por resultados (Ouchi, 1979) deixa em voga a discussão sobre a qualidade dos serviços. Como trabalho futuro ou expansão deste, sugere-se um estudo mais aprofundado das diferenças entre microrregiões sobre a fronteira e aquelas abaixo da fronteira eficiente.

Por fim, o trabalho assevera a importância da utilização de ferramentas de controle baseando-se em resultados para promover políticas para a melhoria da eficiência da saúde, que considerem as diversidades dos municípios e microrregiões do país, em função das particularidades inter-regionais.

\section{Referências Bibliográficas}

Alfradique, M. E. et al (2009). Internações por condições sensíveis à atenção primária: a construção da lista brasileira como ferramenta para medir o desempenho do sistema de saúde (Projeto ICSAP - Brasil). Cadernos de Saúde Pública, 25 (6), p. 8-15.

Anthony, R. N. (1965) Planning and Control Systems: framework for analysis. Boston: Graduate School of Business Administration Harvard University, 1965.

Anthony, R. N. (2002) Govindarajan, V. Sistemas de controle gerencial. São Paulo: Atlas, 2002.

Aubert, B.A. \& Boudeuau, S. (2012) Public sector performance and descentralization of decision rights. Canadian Public Administration, 44 (4).

Banker, R.D., Charnes, A. \& Cooper, W.W (1984). Some models for estimating technical and scale inefficiencies in data envelopment analysis. Management Science, 30 (9), 1078-1092.

Belloni, J.A. (2000) Uma metodologia de avaliação da eficiência produtiva de universidade federais brasileiras. Tese (Doutorado em Engenharia de Produção) - Universidade Federal de Santa Catarina, Florianópolis, SC. 156 p.

Benavent, C. \& Villarmois, O (2006). CRM, apprentissage et controle organisationnel. Journal of Information Systems and Technology Management, 3 (2), 2006, 193-210.

Berry, A. J. \& Broadbent, J.; Otley, D. Management control: theories, issues and performance. 2 ed. New York: Palgrave Macmillan, 2005.

BRASIL. Constituição (1988). Constituição da República Federativa do Brasil. Brasília: Senado Federal, Centro Gráfico, 1998.

Brasil. Ministério da Saúde. Agência Nacional de Saúde Suplementar (2007). Promoção da Saúde e Prevenção de Riscos e Doenças na Saúde Suplementar: manual técnico. $2^{\underline{a}}$ Edição Revisada e Atualizada. Rio de Janeiro: ANS. 168 p.

Bretschneider, S., Ballal, S., Genest, A. \& Smith, S. (2007) Measuring management contribution through decomposition of efficiency scores. 9 th National Public Management Conference, Tucson, Arizona.

Caminal, J. et al. (2004) The role of primary care in preventing ambulatory care sensitive conditions. European Journal of Public Health, 14 (3), p. 5.

Cardoso, C. S. et al. (2013) Contribuição das internações por condições sensíveis à atenção primária no perfil das adminissões pelo sistema público de saúde. Revista Panamericana Salud Publica, 34 (4), p. 8

Castiel, L. D. (2008) Inefetividade e ineficiência: reflexões sobre a epidemiologia e os serviços de saúde de um estado de mal-estar social. Cad. Saúde Pública, 6 (1), Rio de Janeiro, jan./mar.

Charnes, A., Cooper, W.W., Rhodes, E. (1978) Measuring the efficiency of decision-making units. European Journal of Operational Research, 2 (1), 429-444. 
Charnes, A., Cooper, W.W., Lewin, A.Y. \& Seiford, L.M. Data envelopment analysis: theory, methodology, and application. Dordrecht: Kluwer Academic, 1994. $513 \mathrm{p}$.

Chtioui, T., Thiery-Dubuisson, S. (2011) Hard and soft controls: mind the gap. International Journal of Business, 16 (3), 289.

Costa, G.D., Cotta, R.M.M., Reis, J.R., Ferreira, M.L.S.M., Reis, R.S. \& Franceschini, S.C.C. (2011). Avaliação da atenção à saúde da criança no contexto da Saúde da Família no município de Teixeiras, Minas Gerais (MG, Brasil). Ciência \& Saúde Coletiva, 16 (7), 3229-3240.

Czeresnia, D. The concept of health and the difference between prevention and promotion. Cadernos de Saúde Pública, 15 (4), 701-709, 1999.

Datasus. Informações de saúde. Disponível em: <http://www.datasus.gov.br>. Acesso em: 16 fev. 2008.

Drury, C. Management and cost accounting. 6 ed. London: Thomson Learning, 2004.

Emmanuel, C., Otley, D. \& Merchant, K. (editores). Readings in accounting for management control. London: Chapman \& Hall, 1993.

Estelita Lins, M.P. \& Meza L.A. (org.). (2000) Análise envoltória de dados. Rio de Janeiro: COPPE/UFRJ, 2000.

Faria, F. P., Jannuzzi, P. M. \& Silva, S. J. Eficiência dos gastos municipais em saúde e educação: uma investigação através da análise envoltória no estado do Rio de Janeiro. Revista de Administração Pública, 42 (1), p. 155177, jan./fev. 2008.

Fontenelle, L. F. (2011) Estratégia Saúde da Família e Internações por Condições Sensíveis à Atenção Primária (ICSAP): revisão sistemática da literatura. 44f Departamento de Medicina Social da Faculdade de Medicina de Ribeirão Preto, Universidade de São Paulo

Forbes, M \& Lynn, L.E. Governance and Organizational Effectiveness: toward a theory of government performance. Public Services Performance: Perspectives on Measurement and Management, Cambridge, Cambridge University Press, 2007.

Fleury, S.S.B. \& Baris, E (2001). Reshaping health care in Latin America: a comparative analysis of health care reform in Argentina, Brazil, and Mexico. International Development Research Centre (IDRC).

Gimzauskiene, E. \& Valanciene, L. (2010) Efficiency of performance measurement system:the perspective of decision making. Economics and management, 15 (1).

Gomes, J. S. \& Salas, J. M. A. (2001) Controle de Gestão: Uma abordagem contextual e organizacional. $3^{\text {a }}$ Ed. São Paulo: Ed. Atlas.

Gonçalves, A. C., Noronha, C. P., Lins, M. P. E. \& Almeida, R. M. V. R. (2008) Análise Envoltória de Dados na avaliação de Hospitais públicos nas capitais brasileiras. Rev. Saúde Pública, 41(3), p.1-8. 2008. Disponível em: < http://www.scielosp.org/pdf/rsp/v41n3/5327.pdf>. Acesso em: 21 fev. 2008.

Gonçalves, M.A., Santos, L. M., Dias, A. T. \& Ferreira, M.A.M. (2012) Uma Análise da Mudança da Produtividade da Alocação de Recursos Públicos na Atenção Básica da Saúde em Municípios da Região Sudeste Brasileira. Revista de Ciências da Administração (CAD/UFSC), 14, (1), 60-74.

Govindarajan, V. A. (1988) Contingency approach to strategy implementation at the businessunit level: integrating administrative mechanisms with strategy. Academy of Management Journal, 31 (4), 828853.

Hatch, M. J. (1997). Organization theory: modern, symbolic and postmodern perspectives. New York: Oxford University Press.

Henrique, F. \& Calvo, M.C.M. (2009) Grau de implantação do Programa Saúde da Família e indicadores sociais. Ciência e Saúde Coletiva, 14 (1), 1359-1365.

Instituto Brasileiro de Geografia e Estatística (IBGE). (2009) Divisão

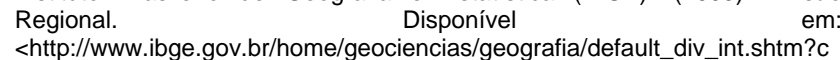
$=1>$. Acesso em: 20 jul.

Lans, L.Q. \& Tomei, P.A. (2014) Confiança versus controle: análise da governança do fundo garantidor para investimentos. Revista Eletrônica de Estratégia \& Negócios, 7 (1)

Marinho, A (2003). Avaliação da eficiência técnica nos serviços de saúde nos municípios do estado do Rio de Janeiro. Revista Brasileira de Economia, 57 (3), Rio de Janeiro, Jul./Set.
Marinho, A. (2008) Estudo de eficiência em alguns hospitais públicos e privados com a geração de rankings. Ministério do Planejamento, Orçamento e Gestão. IPEA. 2001. Disponível em: <http://www.ans.gov.br/portal/upload/forum_saude/forum_bibliografias.pdf >. Acesso em fev. 13 fev. 2008.

Mendes, Á.; Leite, M. G. \& Marques, R. M. (2011). Discutindo uma metodologia para a alocação equitativa de recursos federais para o Sistema Único de Saúde. Saúde e Sociedade, 20 (3), p. 17-25.

Mendes, E.V. (2012) O cuidado das condições crônicas na atenção primária à saúde: o imperativo da consolidação da estratégia da saúde da família. Brasília: Organização Pan-Americana da Saúde.

Menicucci, T. M. G. (2003). Público e privado na política de assistência à saúde no Brasil: atores, processos e trajetória. Tese (Doutorado em Sociologia e Política). Faculdade de Filosofia e Ciências Humanas, Universidade Federal de Minas Gerais.

Mintzberg, H. The structuring of organizations. New Jersey: Prentice Hall, 1979

Mintzberg, H.; Lampel, J.; Quinn, J. B. \& Ghoshal, S. (2006) O processo da estratégia. 4 ed. Porto Alegre: Bookman.

Ouchi, W. G. (1979) A conceptual framework for the design of organizational control mechanisms. Management Science. 25 (9), 833-848.

Rechel, B. \& Blackburn, C. M.; Spencer, N. J.; Rechel, B. (2009) Access to health care for Roma Children in Central and Eastern Europe: findings from a qualitative stud in Bulgaria. International Journal for Equity in Health, 8 (24).

Rizzotto, M. L. F. (2000) O Banco Mundial e as políticas de saúde no Brasil nos anos 90: um projeto de desmonte do SUS. Tese (Doutorado em Saúde Coletiva) - Faculdade de Ciências Médicas. Universidade Estadual de Campinas. Campinas, 2000.

Rosen, M. \& Baroudi, J. (1992) Computer-based technology and the emergence of new forms of managerial control. In: Sturdy, A., Knights, D. Willmott, H. (Eds.). Skill and Consent: Contemporary Studies in the Labour Process. London: Routledge.

Santos, L. M.; Gonçalves, M.A. \& Ferreira, M.A.M. (2014) Performance Evaluation of Expenditure in Primary Care: the case of Brazil s Southeastern Cities. Organizações \& Sociedade (Online), 21, 467-488.

Scott, W. R. \& Davis, G. F. D. (2007) Organizations and Organizing Rational, Natural, and Open System Perspectives. Upper Saddle River, NJ: Prentice Hall, 2007.

Segall, M. (1983) Planning and politics of resource allocation for primary health care: promotions of meaningful national policy. Soc. Sci. Med., 17 (24), p. 13-118.

Selden, S. C. \& Sowa, J. E. (2004) Testing a Multi-Dimensional Model of Organizational Performance: Prospects and Problems. Journal of Public Administration Research and Theory, 14 (3), 395-416.

Serapioni, M. (1999). Avaliação da qualidade em saúde: a contribuição da sociologia da saúde para a superação da polarização entre a visão dos usuários e a perspectiva dos profissionais de saúde. Saúde em Debate, Rio de Janeiro, 23 (53), 81-92.

Starfield, B. (2006) State of the art in research on equity in Health. Journal of Health Politics, Policy and Law, 31 (1), 11-32.

Surco, D.F. (2004) Desenvolvimento de uma ferramenta computacional para avaliação da eficiência técnica baseada em DEA. - Dissertação (Mestrado em Métodos Numéricos em Engenharia) - Setor de Ciência Exatas e Setor de Tecnologia da Universidade Federal do Paraná, Curitiba.

Starfield, B. Shi, L. \& Macinko, J. (2005). Contribution of primary care to health system and health. Milbank Quarterly, 83 (2), 475-502, October 2005. Thiede, M. \& Mcintyre, D. (2008) Information, communication and equitable Access to health care: a conceptual note. Cad. Saúde Pública, Rio de Janeiro, 24 (5), 1168-1173.

World Health Organization. World Health Statistics 2013. Geneva: World Health Organization, 2013.

Zeydan, M. \& Colpan, C (2009). A new decision support system for performance measurement using combined fuzzy TOPSIS/DEA approach. International Journal of Production Research, 47 (15), 4327-4349. 